Milagros Vilar*

\title{
"Hablar en equipo": la construcción de una participación conjunta en reuniones de profesionales de la salud
}

\section{"Speaking as a team": The construction of a joint participation in health professionals' meetings}

https://doi.org/10.1515/soprag-2021-0009

Publicado en línea noviembre 4, 2021

Resumen: La categoría de participación permite observar empíricamente cómo las interacciones sitúan a las personas de maneras específicas en el marco de prácticas sociales concretas. El objetivo de este trabajo es caracterizar el modo en que se organiza la participación en las reuniones de un equipo interdisciplinario de salud en un hospital de Buenos Aires, Argentina. Para ello, analizamos las interacciones orales atendiendo a la manera en que se gestionan los turnos de habla y se interpretan las instancias de habla simultánea en el contexto de dos actividades vinculadas con la toma de decisiones y la resolución de obstáculos en la interacción: una propuesta de escritura y una secuencia de reparación. A través del análisis, definimos la participación conjunta como una forma de participación en la que se prioriza la construcción colectiva por sobre las voces individuales y en la que el habla simultánea tiene naturaleza cooperativa, no disruptiva. Asimismo, identificamos los efectos que produce en las reuniones, orientados a la construcción del consenso, el establecimiento de la continuidad temática y el mantenimiento de las relaciones interpersonales. A la luz de estos resultados, observamos que la participación conjunta tiene un rol central en el proceso de construcción y mantenimiento de una identidad grupal (ser un equipo), reforzando la inscripción de los profesionales en ella.

Palabras clave: participación, análisis conversacional, interacción interprofesional, reuniones de equipo, identidad grupal

\footnotetext{
*Corresponding author: Milagros Vilar, Centro de Estudios del Lenguaje en Sociedad, Universidad Nacional de San Martín, San Martín, Argentina, E-mail: mvilar@unsam.edu.ar
}

כ Open Access. (๑) 2021 Milagros Vilar, published by De Gruyter. (C) BY-NC-ND This work is licensed under the Creative Commons Attribution-NonCommercial-NoDerivatives 4.0 International License. 
Abstract: The category of participation allows us to observe empirically how interactions situate people in specific ways in given social practices. In this article, we aim to characterize how participation is organized in the meetings of an interdisciplinary healthcare team in a hospital in Buenos Aires, Argentina. To this purpose, we analyze oral interactions to explore how turn-taking is managed and how members of this community of practice interpret simultaneous talk in the context of two activities related to decision-making and obstacle resolution in interaction: a writing proposal and a repair sequence. Based on the interactional analysis, we define joint participation as an organization in which collaborative construction is prioritized over individual voices, and in which simultaneous talk is cooperative, not disruptive, in nature. Additionally, we identify the effects that joint participation produces in meetings, oriented towards consensus building, establishment of topical continuity, and the maintenance of interpersonal relationships. Considering these results, we found that joint participation plays a central role in the process of building and maintaining a group identity (being $a$ team), thus enhancing the health professionals' membership within the team.

Keywords: conversation analysis, group identity, interprofessional interaction, participation, team meetings

\section{Introducción}

La "participación" es una noción que refiere tanto a una dimensión de la interacción humana como a una unidad de análisis (Duranti, 2000) y ha sido explorada por diversas corrientes dentro de la lingüística y otras disciplinas como la antropología, sociología o psicología (p. ej. Goffman, 1981; Lave y Wenger, 1991; Goodwin y Goodwin, 2004). En el marco de los estudios sociolingüísticos, tomar la participación como unidad de análisis nos permite establecer conexiones entre las interacciones locales y sus efectos sociales a mayor escala (Rampton, 2017). Al entender los usos lingüísticos como prácticas situadas que intervienen en la producción del orden social, el estudio de la participación nos orienta en la comprensión de los procesos de construcción identitaria que emergen en la interacción (Bucholtz y Hall, 2005).

El propósito de este trabajo es caracterizar ciertas formas de participación que emergen en la interacción de un grupo de profesionales de la salud en un hospital público de Buenos Aires, Argentina. El grupo está compuesto por profesionales de diversas disciplinas, quienes participan semanalmente, desde hace varios años, en un evento al que denominan "reunión de equipo". En este sentido, conceptualizamos al grupo de profesionales como una comunidad de práctica, en tanto 
sus miembros se reúnen semanalmente alrededor de un compromiso mutuo, asociado al desarrollo (y aprendizaje) de una práctica compartida (Eckert y McConnell-Ginet, 1992; Lave y Wenger, 1991).

La pregunta general que guía nuestra investigación es de qué manera la organización de la participación en estas reuniones contribuye a hacer equipo, esto es, de qué manera contribuye (o no) al desarrollo, mantenimiento y adhesión a una identidad grupal (ser un equipo), que los propios actores conciben como un “equipo interdisciplinario”. En este trabajo, argumentaremos que ciertos ordenamientos de la interacción y formas de participación que emergen en las reuniones tienen un rol central en el proceso de conformación y mantenimiento de esta identidad grupal, junto a otros mecanismos que hemos abordado en un trabajo anterior (Vilar, 2020). Así, nos proponemos mostrar cómo, a través de la organización y gestión de la participación en las reuniones de equipo, se construye una participación conjunta que favorece los procesos de afiliación y de construcción de la membresía en esta comunidad de práctica.

El artículo se organizará de la siguiente manera: en el primer apartado, presentamos el marco conceptual y los principales antecedentes del campo en torno a la categoría de participación y el sistema de toma de turnos en la conversación. En el apartado siguiente, introducimos nuestros datos interaccionales y la metodología de análisis. En la tercera sección desarrollamos el análisis, a partir de dos casos seleccionados de nuestro corpus, y, finalmente, en la última sección presentamos los resultados del análisis y su discusión a la luz de los antecedentes del campo.

\section{Marco teórico}

Situamos nuestra investigación en el marco teórico de la sociolingüística interaccional, con una perspectiva etnográfica que posibilita el estudio del lenguaje en una comunidad específica, a partir de las prácticas que la constituyen como tal, y teniendo en cuenta las intuiciones y saberes de los propios actores que la conforman (Codó, Patiño Santos, y Unamuno, 2012; Gumperz, 1982). Desde este marco, la interacción es concebida como el espacio de constitución del orden social, donde tienen lugar los procesos de creación e interpretación de significados, donde se construyen y mantienen las identidades sociales (Gumperz y CookGumperz, 1982). Asimismo, consideramos que estos significados toman forma dentro de relaciones sociales específicas, historias de interacciones y regímenes institucionales, producidos e interpretados por agentes con expectativas y repertorios que deben ser comprendidos etnográficamente (Rampton, Maybin, y Roberts, 2015). 
La noción de participación resulta central en este trabajo, en la medida en que nos permite observar empíricamente cómo las interacciones ubican a las personas entre sí de maneras específicas en el marco de prácticas sociales situadas (Goffman, 1981; Goodwin y Goodwin, 1992). En el caso que analizamos aquí, el estudio de la participación nos permite comprender cómo los profesionales de la salud se posicionan como un equipo cuando realizan ciertas actividades en las reuniones semanales en el hospital. Asimismo, esta categoría se realiza tanto en un nivel micro, en el cual los interlocutores construyen y negocian sus roles y su involucramiento a través de recursos interaccionales específicos, como en un nivel macro, en el que se observan las consecuencias socioculturales de estas acciones y posicionamientos locales (Bucholtz y Hall, 2005, p. 591), que en nuestro análisis orientaremos especialmente a los procesos de construcción de identidades sociales. Así, a través del análisis de la forma en que las personas participan en determinados encuentros y tipos de actividades, podemos estudiar el lugar de la interacción en los procesos de construcción de las relaciones sociales (Goodwin y Goodwin, 2004; Sidnell, 2009).

Al mismo tiempo, pensar en los individuos como participantes nos permite incorporar la totalidad de la experiencia de lo que significa ser miembro de una comunidad y abarcar un conjunto de formas de participación que caracterizan las prácticas comunicativas, que han recibido especial atención por parte de los analistas de la conversación, como el silencio, la escucha activa, el habla simultánea y la producción conjunta de turnos y secuencias interaccionales (Gallardo Paúls, 1993; Lerner, 2000). De este modo, observar las reuniones de equipo haciendo hincapié en la participación permite dar cuenta no solo del modo en que las personas se relacionan entre sí, sino también de las formas en que se vinculan a la comunidad y, al mismo tiempo, hacen comunidad (Duranti, 2000, p. 387). En tal sentido, comprendemos que la participación está en estrecha relación con el establecimiento de la membresía, por lo que puede funcionar como un recurso a través del cual las personas señalan su pertenencia y muestran su afiliación a un grupo (cfr. Bravo, 1999).

Por ello, nos interesa explorar los vínculos entre participación y construcción identitaria, con el fin de comprender de qué modo los profesionales se posicionan como un equipo y llegan a configurar, a través de sus prácticas interaccionales rutinarias, esta identidad grupal (De Fina, 2006; Holmes, 2006). De este modo, buscamos examinar las vinculaciones entre las configuraciones locales de la identidad y las categorías identitarias que circulan en el contexto estudiado, como es el caso de la categoría "equipo interdisciplinario". ${ }^{1}$

1 Se trata de una categoría identitaria que ha sido obtenida a través de la experiencia etnográfica y que nos ha permitido conocer cómo los profesionales de esta comunidad entienden sus propias prácticas, en oposición a otras formas de organizar el trabajo que predominan en la institución hospitalaria. 
Nuestra perspectiva retoma los estudios del análisis conversacional y la lingüística sociocultural sobre la identidad (Antaki y Widdicombe, 1998; Bucholtz y Hall, 2005), que sostienen que las identidades son producidas, actuadas y encarnadas a través de una variedad de recursos en la interacción. Desde este marco, la identidad no es algo que los hablantes "poseen" ni puede ser definida $a$ priori a partir de ciertas categorías sociales, sino que se realiza de manera intersubjetiva en la interacción (Bucholtz y Hall, 2005, p. 587). Por ello, hablamos de "hacer equipo" para referirnos a un conjunto de prácticas interaccionales que se orientan a construir y señalar esta identidad grupal. En este trabajo, ponemos el foco en la manera en que se gestiona la participación y se co-construyen los turnos y secuencias de habla, ya que consideramos que constituyen medios a través de los cuales los profesionales de la salud actúan esta identidad como equipo (cfr. Djordjilovic, 2012) en las reuniones semanales en el hospital.

\subsection{Cómo se organiza la participación}

En uno de los trabajos más conocidos del Análisis de la Conversación, Sacks, Schegloff, y Jefferson (1974) presentan un modelo que establece una sistematización para la organización de la toma de turnos en la conversación, a partir de la postulación de una serie de componentes y reglas. Si bien este modelo contempla la posibilidad de que dos o más personas hablen a la vez, las formas de habla simultánea se consideran breves o excepcionales y se asume que los propios participantes resolverán esos "problemas" en el menor tiempo posible, con el objetivo de restablecer la organización interaccional de "un hablante a la vez". En un trabajo posterior, Schegloff (2000) se refiere a situaciones en que se genera una "pelea por el terreno" (floor fight), en la cual los hablantes tienden a hablar más alto para "ganarle" a otros (Schegloff, 2000, p. 12).

Posteriormente, varios estudios han mostrado la inadecuación de este modelo para dar cuenta satisfactoriamente de lo que sucede en algunas conversaciones con múltiples participantes, en las que entra en juego una dinámica más colaborativa para la construcción y distribución de los turnos (Coates, 1997; Edelsky, 1981; Eppler, 2009; Lerner, 2002). El rasgo competitivo que se instala en la descripción de Sacks et al. (1974) no resulta productivo para describir formas de organización de la participación en las que no es tan fácil determinar a quién le "pertenece" el turno, o instancias de habla simultánea que no se interpretan como amenazas sino como acciones colaborativas (cfr. Gallardo Paúls, 1993) que llevan adelante los participantes en el marco de actividades compartidas.

En su estudio de las reuniones de un grupo de profesores, Edelsky (1981) da cuenta de que existe otro tipo de organización interaccional, al que denomina 
“terreno colaborativo", ${ }^{2}$ en el cual la práctica de hablar simultáneamente es considerada normal. En estos casos, los participantes suelen desarrollar una idea juntos, producir una respuesta conjunta a una pregunta o expresar entre varios una broma, entre otras posibles acciones. En lugar de concebirse como un desvío o un intento por "ganar" el turno de otra persona, el habla simultánea se entiende como un trabajo conjunto para producir significados compartidos, que puede señalar incluso una posición de escucha activa e involucramiento aún mayor por parte de los participantes hacia la actividad en curso (cfr. Coates, 1997).

Sobre la base de estos antecedentes, recuperamos aquí la noción de terreno para explorar las formas de organización de la participación que se llevan a cabo localmente y de manera flexible, como un conjunto de derechos para tomar la palabra vinculados a la actividad en curso (Jones y Thornborrow, 2004). Así, el análisis de la gestión del terreno permite observar cómo se distribuyen estos derechos básicos en una comunidad (Jenks, 2007), atendiendo al modo en que se asignan los turnos de habla y a los significados que se atribuye al habla simultánea y a otras formas en que las personas participan en la interacción.

Si bien se ha analizado la organización de la participación de manera extensa en ciertos eventos y/o actividades, especialmente vinculados a las situaciones de clase (p. ej., Seedhouse, 2004; Jones y Thornborrow, 2004; Moore y Nussbaum, 2013) o a la conversación informal en grupos de mujeres (p. ej. Coates, 1997; Eppler, 2009), más recientemente ha comenzado a analizarse en otras interacciones que involucran a un gran número de participantes, como son las reuniones en distintos ámbitos (Djordjilovic, 2012; Ford, 2008; Markaki y Mondada, 2012). Nuestro trabajo busca contribuir a esta línea de investigación que ha sido poco explorada en el ámbito hispánico, mediante la indagación de las formas de participación en las reuniones de equipos interdisciplinarios en el ámbito sanitario.

\section{Descripción de los datos y marco metodológico}

El presente trabajo es parte de una investigación mayor que estudia la comunicación interprofesional y los mecanismos interaccionales que contribuyen a hacer equipo en las reuniones de un grupo de profesionales de la salud en un hospital público de Buenos Aires (Argentina). ${ }^{3}$ En este marco, los datos fueron recolectados durante un proceso de trabajo de campo que se extendió desde abril de 2017 a enero de 2018, en el que se observaron las reuniones semanales y otras actividades en las que participaban los integrantes del equipo de salud.

2 Del inglés, collaborative floor (mi traducción).

3 Se trata de la investigación que dio lugar a la tesis doctoral de la autora. 
El corpus está constituido por las interacciones orales que tuvieron lugar en treinta y tres reuniones de equipo, lo que alcanza una duración total de 42 horas y 45 minutos de audiograbación. El objetivo principal de este evento es compartir información y acordar líneas de acción conjunta para la atención de pacientes con enfermedades crónicas. Participan entre 8 y 17 profesionales de la salud de diferentes disciplinas (médicas, enfermeros/as, nutricionistas, trabajadoras sociales, psicólogas y una terapista ocupacional), con trayectorias profesionales $\mathrm{y}$ académicas heterogéneas. Para esta investigación, todos ellos firmaron un documento de consentimiento informado en el que expresaron su conformidad a participar. A fin de mantener la confidencialidad de los datos, en la transcripción se han reemplazado los nombres de todas las personas para garantizar el anonimato de los participantes y se han modificado los nombres de instituciones, áreas o consultorios para evitar su identificación.

El abordaje que proponemos para el estudio de la interacción incorpora un repertorio de categorías descriptivas provenientes del análisis de la conversación (Liddicoat, 2007; Sidnell y Stivers, 2013). Siguiendo este marco metodológico, abordamos las reuniones de equipo como eventos interaccionales organizados, lo que supone analizar su estructura interna y dar cuenta de las unidades interactivas que la componen, en términos de actividades, secuencias y turnos de habla. Asimismo, buscamos identificar cuáles son las acciones que los participantes están realizando, o que los demás participantes reconocen que están realizando, y los efectos que dichas acciones producen en la estructura secuencial de la interacción (Heritage y Clayman, 2010, p. 14). Al mismo tiempo, consideramos los efectos sociales de estas acciones, que refieren al impacto en el clima socioemocional de la interacción (Bravo, 2002; Hernández Flores, 2013) y, por lo tanto, en las relaciones interpersonales.

En términos analíticos, proponemos dos orientaciones complementarias para el estudio de la participación en esta comunidad de práctica. Una primera orientación tiene en cuenta principalmente los mecanismos mediante los cuales se distribuye la palabra en las reuniones de equipo, esto es, analiza el sistema de alternancia de turnos y la construcción de las unidades interaccionales. Una segunda orientación toma en cuenta la manera en que los participantes comprenden e interpretan los fenómenos de habla simultánea, tales como solapamientos, interrupciones, completamientos y coproducciones, de acuerdo a los tipos de actividades y sus diferentes grados de involucramiento en ellas. En conjunto, ambas orientaciones permiten abordar la complejidad del fenómeno a estudiar, al dar cuenta de los diferentes aspectos y dimensiones que están implicados en el proceso de participar (y ser participante) en esta comunidad.

A partir de estas orientaciones, en el análisis nos centraremos en ciertos contextos interaccionales que habilitan la construcción de terrenos colaborativos 
en las reuniones de equipo. En particular, observaremos una organización de la participación que denominamos participación conjunta, que refiere a un modo colaborativo de construir los turnos y secuencias de habla y que supone una interpretación del habla simultánea en términos de apoyo (en lugar de competencia). En esta forma de participación, por momentos no resulta sencillo determinar a quién le pertenece el turno (o quién tiene el terreno), en tanto se favorece la construcción conjunta entre varias personas: se genera, así, el efecto de que quien habla es "el equipo" y no un profesional u otro.

Para ilustrar esta forma de participación, presentaremos aquí dos ejemplos que se desarrollan en el marco de dos actividades que se realizan a menudo en las reuniones: en primer lugar, observaremos cómo se actúa esta identidad como equipo en una secuencia de toma de decisiones y, en segundo lugar, cómo se orientan los profesionales para resolver un malentendido en la interacción, a través de una secuencia de reparación que se construye colaborativamente. Considerando otros estudios que han analizado la participación y la construcción de secuencias interaccionales colaborativas en el contexto de narraciones o explicaciones (p. ej., Lerner, 1992; Djordjilovic, 2012; Holmes, 2006), nos interesa avanzar en el análisis de otras actividades en las cuales emergen estas formas de participación conjunta y explorar su relación con los procesos de construcción de una identidad como equipo.

\section{Análisis}

En las reuniones de equipo los desplazamientos en el terreno son frecuentes y se vinculan a los distintos tipos de actividades que allí se realizan. Al mismo tiempo, estos se asocian al hecho de que el sistema de toma de turnos no está establecido de antemano (turn-type pre-allocation system) ni tampoco organizado por una persona dedicada exclusivamente a tal fin (mediated turn taking system), que son las formas más frecuentes de gestionar la participación en interacciones grupales en entornos institucionales (Heritage y Clayman, 2010). En esta comunidad, la participación se gestiona localmente y se organiza según la común interpretación de lo que está sucediendo, como mostraremos en los apartados que siguen.

\subsection{Tomar decisiones en equipo: la construcción del consenso a través de una actividad de escritura}

El primer fragmento que analizamos corresponde a una reunión en la cual se estaba tratando el tema de un paciente que el equipo había decidido internar por 
un motivo social, a raíz de una situación de desprotección familiar. Transcurridas varias semanas de internación, en sucesivas reuniones los profesionales habían estado discutiendo los criterios para determinar el alta del paciente. En la reunión \#12 observamos un momento de la discusión, que llevaba ya unos veinte minutos, en el cual la trabajadora social (JUL) ${ }^{4}$ plantea la necesidad de establecer ciertos objetivos claros y medibles que deban cumplirse como condición para el alta. En ese punto, una de las médicas (ANA) expone cuáles son esos objetivos que deberían plantearle a la familia del paciente:

(1)

Fragmento 1 (parte 1$)^{5}$

Reunión \#12. Cantidad de participantes en total: 10.

1 ANA: [PARA MÍ | son] cuatro cosas $\mid$ digo=

2 JUL: $=$ dale $\mid$ [janotemos!]

3 ANA: [cuatro] cosas| que-

4 que uno puede plantear

5 JUL: ((asiente))

6 ANA: una que- >que santiago tenga su

7 tratamiento una vez por semana acá<।

8 que la madre tenga una vez por semana

9 tratamiento psicológico | que el padre

10 venga a entre[narse]

11 CLA: $\quad\left[{ }^{\circ} \mathrm{a}\right.$ entrenar $\left.{ }^{\circ}\right]$

12 ANA: y $\uparrow$ que: e: la alimentación del

13 chico la maneje la madre

14 JUL: ${ }^{\circ}$ bien $^{\circ}$

Este fragmento muestra la iniciativa llevada adelante por dos participantes (ANA y JUL) de resolver una cuestión que venía discutiéndose desde hacía varias reuniones. La resolución implica llegar a un acuerdo acerca de cuáles son las condiciones para el alta del paciente, a quien hemos llamado Santiago. En este punto, ANA inicia una secuencia que, si bien se introduce como una opinión personal ("PARA MÎ"), funciona como una síntesis de lo conversado hasta el momento por el equipo, que queda reducido en una enumeración de cuatro condiciones que la familia deberá cumplir.

4 Todos los nombres que se mencionan, tanto de profesionales como de pacientes, han sido modificados.

5 Debido a la extensión del fragmento, lo presentamos en dos partes, para favorecer la claridad del análisis. Las convenciones adoptadas para la transcripción se especifican en el Apéndice de este trabajo. 
La introducción realizada por ANA (1.1) motiva en JUL la propuesta de registrar por escrito aquello que se anuncia como una enumeración (“cuatro cosas"): “ianotemos!” (1.2). Así, a partir de la propuesta de JUL, el marco de la actividad se modifica: deja de ser una formulación de síntesis individual para transformarse en una actividad de escritura, una instancia que propicia la participación de un número mayor de personas. En efecto, durante la exposición de ANA de los cuatro puntos, otras dos participantes (JUL y CLA) realizan acciones de afiliación y alineamiento (cfr. Bonnin, 2019), en las que expresan su acuerdo y la alientan a continuar (1. 5, 11 y 14).

Hacia el final, la trabajadora social (JUL) inicia un movimiento de recapitulación de lo expresado por su colega, necesario para ratificar y completar sus anotaciones, tal como vemos en el fragmento que sigue: ${ }^{6}$

\section{Fragmento 1 (parte 2)}

29 JUL: dijimos $\downarrow$ | que venga santiago

30 una vez por semana=

31 ANA: $=$ sí=

32 JUL: $=$ a tratamiento $\downarrow$ que venga la-

33 que la mamál [e: haga]

34 VAL: [haga [su tratamiento $\left.]_{2}\right]_{1}$

35 LUZ: [venga $]_{2}$

36 CLA: [venga a terapia $]_{2}$

37 JUL: $\quad[\text { (inaud.) }]_{2}$ una vez por se[mana]

38 ANA: [Y QUE] | nos dé- $\mid$ o sea nos dé el

39 contacto con la psicó:loga=

40 JUL: =sí

41 ANA: que- que la psicóloga acá-

42 el equipo de salud se pueda poner

43 en contacto con la psicóloga que

44 va a seguir a la madre de santiago

45 JUL: que la madre administre | e:

46 VAL: el dinero

47 JUL: el dinero | para la compra de:

48 ANA: de alimentos | por todo lo que ha-

49 ha venido suce[diendo]

50 VAL: [sí]

6 Por cuestiones de foco, se han omitido algunas líneas de la transcripción entre ambos fragmentos. 


\begin{tabular}{|c|c|c|}
\hline 51 & & $<2>$ \\
\hline 52 & ANA: & ¿y? | la otra \\
\hline 53 & & [era: que [el padre| venga $\left.]_{2}\right]_{1} \mid$ \\
\hline 54 & & a entrenarse \\
\hline 55 & CLA: & {$[>\text { el entrenamiento del padre }<]_{1}$} \\
\hline 56 & NES: & [(inaud.) del padre $]_{2}$ \\
\hline 57 & & $<7>$ \\
\hline 58 & ANA: & en el tratamiento de su hijo | \\
\hline
\end{tabular}

En esta segunda parte, observamos que JUL, quien había propuesto tomar nota de los criterios acordados (1. 2), inicia una secuencia de recapitulación que resulta clave, en tanto le confiere un carácter colectivo a lo dicho: el uso de la primera persona del plural (“dijimos”, 1.29) presenta los cuatro puntos mencionados como el resultado de una discusión del equipo, y no como la decisión de una de sus integrantes (cfr. Mondada, 2015; Hall y Butler, 2017). Al mismo tiempo, la acción de recapitulación y posterior registro escrito hace público el proceso de reconstrucción de las decisiones tomadas, producto de discusiones que sucedieron en varias reuniones, y permite que los demás participantes monitoreen qué es lo que está siendo registrado, lo corrijan o expresen su desacuerdo. En este marco, JUL adopta el rol de portavoz, al comunicar (y registrar por escrito) una decisión cuya autoría se presenta como colectiva.

Así, se inicia una secuencia extensa de recapitulación (1.29 a 58), en la que se construye un terreno colaborativo que habilita la participación de un número mayor de personas (6 de los 10 profesionales presentes aquel día) para la realización de una actividad conjunta. Allí observamos una serie de rasgos que caracterizan a la participación conjunta: en primer lugar, la presencia de turnos encadenados, esto es, turnos realizados por diferentes personas, entre los cuales no se registra una pausa audible. Este encadenamiento de turnos señala un compromiso mutuo y activo para llevar a cabo la actividad en curso: a partir de réplicas breves que se producen inmediatamente a continuación (o, en algunos casos, superpuestas al final de un turno) se alienta a seguir adelante, como se puede observar en los turnos sucesivos de JUL y ANA (1.29-33 y 38-40) y ANA y VAL (1.48-50).

En segundo lugar, la participación conjunta se observa en la terminación de enunciados anteriores no finalizados. En este fragmento observamos una secuencia de turnos colaborativa (Lerner, 2000) o producción colaborativa (Szczepek, 2000), mediante los enunciados de completamiento (utterance completions). En estos turnos se observa la colaboración de dos (o más) hablantes para producir una única unidad sintáctica, en el sentido de que el siguiente hablante anticipa y produce la finalización de una unidad de turno iniciada por un hablante 
anterior. En nuestro caso, el turno iniciado por JUL (“que venga la- que la mamá [e: haga]”, 1.32-33) se superpone con los de VAL, LUZ y CLA, quienes ofrecen terminaciones posibles para el enunciado anterior: "haga su tratamiento" (1.34), “venga” (1.35) y "venga a terapia” (1.36).

Los completamientos del enunciado de JUL se producen luego de una interrupción en el habla, una pausa y un alargamiento vocálico, rasgos prosódicos que son indicadores de una invitación a participar. Si bien el turno de JUL queda incompleto, ella llega a producir parte de la terminación ("haga”), en simultáneo con las demás personas, tras lo que se interrumpe, permitiendo que los demás se expresen. Finalmente, ella ratifica y expande los enunciados anteriores, agregando información acerca de la frecuencia del tratamiento: "una vez por semana" (1.37). En ninguno de estos casos las superposiciones son interpretadas como interrupciones, ${ }^{7}$ pese a realizarse en instancias de la unidad de turno que no constituyen lugares apropiados para la transición. En cambio, son interpretadas como acciones colaborativas, a veces promovidas por los mismos participantes, como lo señalan los rasgos prosódicos observados y las acciones posteriores que suscitan.

Este mismo tipo de secuencia colaborativa se produce más abajo, cuando JUL deja en suspenso el enunciado: “que la madre administre | e:” (1.45). La extensión vocálica, luego de una pausa breve, puede ser interpretada como un pedido de asistencia o elicitación de información para la tarea de escritura que está realizando, por lo que la médica (VAL) a continuación ofrece una respuesta ("el dinero", 1.46). En este caso, JUL responde a esta terminación propuesta: en señal de conformidad, la repite y continúa su turno hasta un próximo punto en que se detiene tras un nuevo alargamiento vocálico (“el dinero | para la compra de:”, 1.47). En esta instancia, es ANA quien ofrece una propuesta de finalización para esta unidad sintáctica: “de alimentos” (1.48). A continuación, tras una pausa breve en la que nadie toma el turno, pese a que la unidad de turno ha sido completada, ANA lo expande mediante una justificación.

Finalmente, luego de una pausa de dos segundos, ANA toma el turno para retomar la recapitulación iniciada por JUL para la actividad de escritura, con el último de los cuatro puntos. Al igual que en los turnos de JUL, al producir un alargamiento vocálico en mitad de la unidad de turno, junto con una entonación interrogativa en el inicio (“¿y? | la otra era:”, 1.52-53), otros participantes producen enunciados breves de completamiento, que se realizan de manera simultánea a la

7 A diferencia de lo que sucede en otros contextos conversacionales, aquí no aparecen rasgos que indiquen esta interpretación, como podrían ser pedidos explícitos de que otros abandonen el terreno, que hemos encontrado en otros contextos, como los episodios de desacuerdo ("dejame hablar a mí”, "pará”, “dejame terminar”). 
finalización del turno realizada por ANA. En este caso, una de las enfermeras (CLA) propone una terminación posible (">el entrenamiento del padre<", 1.55), cuya formulación difiere en su estructura sintáctica con la producida por ANA ("que el padre venga a entrenarse”, 1.53-54), y el enfermero (NES) colabora con otra opción que, al superponerse, no llega a ser completamente audible pero aparenta ser muy similar ("(inaud.) del padre”, 1.56).

La pausa extensa que sigue señala el compromiso del resto de los participantes en la actividad en curso, quienes dejan de hablar mientras JUL está escribiendo (1.57). A continuación, ANA vuelve a intervenir con un turno que muestra su subordinación (en términos sintácticos y semánticos) con los anteriores, en tanto retoma el último punto y lo completa, explicitando a qué se refieren con "entrenamiento" (1.58).

De este modo, observamos cómo una propuesta de escritura permite la construcción de la participación conjunta, a partir de la cual muchos de los integrantes del equipo realizan contribuciones y son invitados a participar para alcanzar un propósito común. Si bien la actividad de escritura no implica a priori una actividad colaborativa, hemos visto cómo en este caso se desarrolla colaborativamente, a través de una secuencia de recapitulación en la que las voces individuales se unen para producir una voz colectiva que expresa la decisión tomada por el equipo. Así, podemos observar cómo los recursos que despliegan los participantes para la gestión y construcción de turnos contribuyen a hacer relevante una identidad grupal; en otras palabras, cómo, a través de su participación, los profesionales actúan como un equipo y, con ello, logran un mayor involucramiento y cohesión para alcanzar una decisión adecuada. En cuanto a los efectos que produce, hemos mostrado cómo esta organización de la participación permite resolver un tema que se presentaba complejo y avanzar, así, hacia los objetivos de la reunión. Asimismo, genera un impacto positivo en las relaciones interpersonales ya que, como hemos visto, permite construir el consenso de los miembros del equipo, al involucrarlos y hacerlos partícipes de la decisión tomada.

\subsection{Resolver un malentendido en la interacción: las acciones de reparación colaborativa}

Otros contextos en los que se propicia la participación conjunta en las reuniones son aquellos en donde uno o más participantes perciben un obstáculo en la interacción, esto es, reconocen algo que amenaza o imposibilita la comprensión, y producen una secuencia de reparación orientada a resolverlo. A diferencia del fragmento anterior, aquí la participación conjunta emerge en una secuencia 
incrustada breve que cumple la función de gestionar localmente lo que se concibe como un problema: en este caso, un malentendido que interrumpe la continuidad de la actividad que se estaba realizando (el informe sobre una paciente):

\section{Fragmento 2}

Reunión \#4. Cantidad de participantes en total: 10.

1 VAL: (...) e:: en cuanto al peso | ella ayer vino ta-

2 había pesado el nueve seis tres veinte y ayer pesaba-

3 LEO: no: seis

4 ANA: SE::IS=

5 MAY: =seis

6 VAL: seis tres vein[te]

7 MAY: $\quad$ [el] nueve [>quiso decir<]

8 ANA: [no $\mid$ nueve no]

9 MAY: el nueve de ma[yo]

10 ANA: [seis]

11 MAY: pesó seis

12 ANA: dijiste [nueve]

13 VAL: [el $\uparrow$ nueve]

14 FLO: el nue[ve de mayo]

15 VAL: [el nueve] / de mayo

16 ANA: $[\uparrow \mathrm{A}:::::]_{1}$

17 MAY: [no | que el nueve] $]_{1}$ pesó

18 [seis tres veinte] ${ }_{2}$

19 ANA: [ ${ }^{\circ}$ yo entendí ${ }_{2}$

20 VAL: seis tres veinte | [y: ayer $]_{3}$

21 pesaba seis cuatrocientos $\langle 2>$

22 bueno s- e: sigue sin la so::nda |

$23 \quad{ }^{\circ}$ bueno $^{\circ \circ}<2>$ [hoy tiene- $]_{4}$

24 ANA: $\quad[(\text { (risas })]_{3}$

25 MAY: [estuvo con] $]_{4}$ menos vómitos

En este fragmento se observa la emergencia de un obstáculo en la interacción, causado por un malentendido que se genera entre varios participantes. Una de las médicas (VAL) estaba comunicando la evolución de una paciente, señalando la fecha y el peso, cuando el enfermero (LEO) interviene para corregir el dato del peso (1.3). El malentendido se debe a que, al pronunciar varios números sucesivamente sin realizar ninguna pausa entre ellos ("nueve seis tres veinte", 1.2), algunos de los profesionales comprenden erróneamente el enunciado de la médica, 
confundiendo la información que refiere a la fecha ("el nueve") y al peso ("seis tres veinte").

La reparación es un mecanismo gestionado localmente y organizado interaccionalmente a través del cual los participantes buscan preservar la comprensión intersubjetiva (Schegloff, 1992) y, en tal sentido, se caracteriza por tener una implicación colaborativa, ya que se privilegia la finalidad de los participantes de entenderse y de colaborar en la interacción para lograr ciertos objetivos (Acosta, 2017). También se han documentado otros usos interaccionales de la reparación, más allá de la gestión de obstáculos que emergen en el habla; algunos estudios han asociado esta práctica con la expresión del desacuerdo y la desafiliación (Hayashi, Raymond, y Sidnell, 2013; Pomerantz, 1984) y se ha observado que, en ocasiones, funciona como un recurso que permite gestionar el conflicto en la interacción (Glenn, 2019; Kitzinger, 2012), al tratarlo como si fuera un problema de comprensión (Schegloff, 2007, p. 151).

Típicamente las secuencias de reparación se organizan en un par adyacente que consta de dos partes: una primera parte en la cual se identifica la fuente del problema, y una segunda parte en la cual se propone una solución para este. En el fragmento analizado, la secuencia de reparación es iniciada por un participante diferente a quien ha producido la fuente de disturbio. LEO señala el problema inmediatamente después de haber finalizado la unidad de construcción de turno de VAL, dejando interrumpida la siguiente unidad de turno que ya había iniciado ("y ayer pesaba-", 1.2). La manera de gestionar la reparación consiste, en este caso, en el señalamiento de un error ("no") y, luego, en el ofrecimiento de una propuesta de reformulación ("seis"), que constituye la reparación propiamente dicha (en este caso, una corrección).

A diferencia de otros tipos de reparación, en los que se da lugar al hablante que produce la fuente de disturbio la tarea de solucionarlo, en este caso ambas acciones convergen en un mismo participante, por lo que se trata de una heterorreparación heteroiniciada (other-initiated other-repair) según la clasificación formulada por Schegloff, Jefferson, y Sacks (1977). De acuerdo a estos autores, la heterorreparación suele ser la opción no preferida en el habla informal, por lo que a menudo es evitada o realizada de manera encubierta, de modo de cuidar la imagen del participante que realizó el turno reparable. Asimismo, suele asociarse a situaciones en que se presenta una asimetría entre los participantes, como los ámbitos de aprendizaje, en los que se ha estudiado extensamente (cfr. Acosta, 2017).

En el caso analizado, observamos que el enfermero (LEO) no atenúa este acto de reparación sino que lo realiza de manera directa. Incluso aquí la corrección es reiterada luego por otros, lo que da lugar a una acción de reparación llevada adelante por varios participantes: la otra médica (ANA) muestra su afiliación con 
LEO, y en un volumen de voz más elevado repite enfáticamente la corrección realizada (1.4), a la cual le sigue otra repetición realizada por una de las nutricionistas (MAY) inmediatamente después. De este modo, los movimientos de tres participantes convergen para reparar el turno de otro participante, mediante una corrección que no es interpretada aquí como un acto descortés hacia VAL, ni como una interrupción que intenta disputar el terreno, sino como una acción que busca continuar con la actividad, privilegiando los propósitos y necesidades del grupo. ${ }^{8}$

En general, cuando la reparación es realizada por una persona diferente a la que produjo el turno que resultó problemático, se espera que el primer hablante responda a la reparación; en este caso, que VAL acepte o rechace la corrección realizada por sus colegas. Sin embargo, al haber pronunciado el mismo número inicialmente, VAL no parece interpretarla como una corrección y, por lo tanto, no responde a ella de la manera esperada (no la acepta ni rechaza). En cambio, ofrece una nueva repetición, expresando el número completo que hace referencia al peso de la paciente (1.6), como un modo de superar el obstáculo y seguir adelante. Con este movimiento, se alinea a las acciones previas y se convierte, así también, en parte realizadora de la reparación.

En un intento por señalar el motivo del malentendido y proteger la imagen de VAL, la nutricionista (MAY) interviene para explicar que el número nueve hace referencia a la fecha, no al peso del paciente: "el nueve >quiso decir<" (1.7), "el nueve de mayo" (1.9). Sin embargo, su intento de explicación queda trunco porque ANA interviene para corregir nuevamente esta cifra y los enunciados de ambas se superponen (1.7-10). Entonces ANA se dirige directamente a VAL, quien produjo la fuente de disturbio originalmente, retomando sus palabras: “dijiste nueve” (1.12). En respuesta, la médica reitera este número, sin explicitar cuál es el motivo de la confusión, pero incorporando un determinante que da cuenta de que se trata de la fecha y no del peso: “[el $\uparrow$ nueve]” (1.13).

A continuación, otra de las nutricionistas (FLO) interviene para completar el enunciado con el mes ("el nueve de mayo", 1.14), en una orientación similar a la de MAY, a fin de evitar que el malentendido siga extendiéndose. En un turno que se superpone parcialmente, VAL repite las palabras de FLO y menciona el mes, por lo que enseguida ANA emite una vocalización que da cuenta de que comprendió el origen del problema (1.16). La risa de ANA, finalmente, funciona como estrategia para resguardar su imagen frente a una situación embarazosa, tras comprender que fue ella la responsable del malentendido.

8 Cabe destacar que, al tratarse de un informe médico sobre un paciente, el señalamiento y la resolución del malentendido resultan acciones de suma relevancia para las tareas del equipo de salud, en tanto podría derivar en errores en la atención. En tal sentido, la reparación es tratada aquí como una acción que involucra al grupo de profesionales en tanto equipo. 
Este fragmento muestra un episodio de resolución de un malentendido, en el que intervienen varios participantes en un esfuerzo conjunto por superar un obstáculo interaccional. En este marco, se habilita un terreno colaborativo que propicia la participación conjunta en una secuencia de reparación construida de manera colectiva, en la que varios participantes se unen para solucionar un problema local que interrumpe la continuidad temática de la reunión. Así, los rasgos que asume la participación (en este caso, los numerosos solapamientos, los turnos encadenados y las repeticiones de enunciados breves) y las repetidas reformulaciones que se suceden para aclarar el malentendido (señaladas mediante expresiones metacomunicativas, como "quiso decir" o "dijiste" en las líneas 7 y 12) ponen de manifiesto que la resolución se concibe como una acción colectiva. En este sentido, vemos que intervienen más participantes que los que habían iniciado la reparación (LEO y ANA) y que, pese a ser señalado como un "error" en el turno de VAL, ese error es asumido de manera colectiva. La acción de atender el obstáculo y solucionarlo compromete a varios participantes, quienes se alinean como equipo para restablecer la intercomprensión y avanzar en las actividades en el marco de la reunión, logrando recomponer la continuidad temática y, al mismo tiempo, cuidar las relaciones interpersonales.

De este modo, la participación conjunta habilita una resolución colectiva y permite que VAL siga adelante con el informe del paciente: al resolverse el malentendido, se cierra una secuencia incrustada y la médica continúa con el informe, justo en el punto en que lo había abandonado por la reparación ("y ayer pesaba" se reitera en 1.2 y 1.20-21). A continuación, vemos que el terreno, que se había ampliado a múltiples participantes en simultáneo, vuelve a modificarse (en este caso, a reducirse) de acuerdo con los requerimientos de la actividad.

\section{Conclusiones}

En este trabajo nos propusimos caracterizar las formas de participación conjunta que emergen en las reuniones de profesionales de la salud en una comunidad de práctica. Para concretar este objetivo, realizamos un análisis de los mecanismos de toma de turnos, los fenómenos de habla simultánea y la construcción de las unidades de habla en el contexto de dos actividades vinculadas a la toma de decisiones y la resolución de obstáculos en la interacción. De este modo, observamos cómo se logra gestionar un terreno conversacional colaborativo, en el que el acceso a la palabra no se presenta restringido ni mediado y en el cual la práctica de hablar simultáneamente es considerada normal.

A partir del análisis de dos ejemplos de nuestro corpus, ilustramos una forma particular de participación que denominamos participación conjunta, que puede 
describirse a partir de una serie de características que hemos señalado en la interacción: se desarrollan turnos más cortos, los enunciados son construidos conjuntamente, se produce una gran cantidad de solapamientos o turnos encadenados, enunciados de completamiento, repeticiones de palabras o enunciados, y la ocurrencia simultánea de enunciados breves (en coro) o risas, entre otros fenómenos. Además, en esta organización de la participación, observamos que el habla simultánea no tiene naturaleza disruptiva, sino cooperativa: es esperable que se produzca y da cuenta del compromiso y el involucramiento activo de los participantes en la acción conjunta. A diferencia de otras formas de organizar la interacción en reuniones, en que se concede un acceso prioritario al terreno a un único participante (cfr. Ford, 2008), en el análisis identificamos dinámicas interaccionales similares a las referidas en los trabajos de Edelsky (1981), Coates (1997) y Eppler (2009).

Asimismo, el análisis nos permitió identificar cuáles son las funciones que cumple la participación conjunta y qué efectos produce esta organización de la participación en el contexto de las reuniones. En primer lugar, encontramos que el establecimiento de un terreno colaborativo a partir de una propuesta de escritura funciona como estrategia para presentar una decisión como compartida y alcanzar el consenso sobre una acción futura. En este caso, la participación conjunta no solo es motivada por la actividad de escritura sino que se sostiene en la interacción a través de continuas elicitaciones y pedidos de asistencia, como también ha documentado Szczepek (2000, p. 25). Estos hallazgos nos permiten advertir que el resultado alcanzado en la reunión (por ej., una decisión o un acuerdo) no es casual, sino que es producto de acciones, muchas veces deliberadas, que llevan a cabo los participantes en la interacción.

En segundo lugar, observamos que la participación conjunta se desarrolla en el marco de las acciones de reparación, mecanismos conversacionales que funcionan para superar obstáculos locales en la interacción. En particular, observamos cómo la participación conjunta da cuenta de un esfuerzo compartido por resolver un malentendido que emerge en la interacción, permitiendo conservar tanto la intercomprensión como las relaciones interpersonales que se requieren para sostener la práctica de esta comunidad. En este sentido, hemos mostrado que los efectos de la participación conjunta se vinculan no solo a los resultados de la actividad (la búsqueda del consenso, la resolución de un malentendido) y de la reunión en general (el tratamiento de los temas establecidos en la agenda) sino también al cuidado y mantenimiento de las relaciones entre los miembros de esta comunidad de práctica.

Finalmente, nos interesa mencionar las vinculaciones entre las dinámicas interaccionales locales y sus efectos a mayor escala; en particular, observar el modo en que la gestión de la participación se vincula con la construcción y 
mantenimiento de una identidad grupal que es aludida constantemente en esta comunidad de práctica: el equipo interdisciplinario. Considerando que la participación constituye una manera de actuar las identidades en la interacción, concluimos que la participación conjunta es un mecanismo mediante el cual los profesionales hacen equipo, es decir que les permite orientarse hacia esta identidad grupal y reforzar su pertenencia en ella. En tal sentido, el análisis detallado de las formas de organizar y gestionar la participación conjunta en las reuniones nos ha permitido no solo caracterizar los recursos interaccionales que posibilitan la construcción de un terreno colaborativo, sino también indagar en cómo los posicionamientos locales impactan en procesos de construcción identitaria a mayor escala.

\section{Apéndice: convenciones de transcripción}

$\begin{array}{ll}>< & \text { ritmo acelerado } \\ <> & \text { ritmo lento } \\ - & \text { corte repentino, interrupción } \\ { }^{\circ} \text { palabra } & \text { pronunciado en voz baja } \\ \text { MAYÚS } & \text { pronunciado en voz alta } \\ ¿ ? & \text { entonación interrogativa } \\ i ! & \text { entonación exclamativa } \\ \uparrow \downarrow & \text { tono ascendente o descendente } \\ \text { a: a:: a::: } & \text { alargamiento de un sonido (de menor a mayor duración) } \\ \text { | } & \text { pausa breve } \\ <2> & \text { pausas cronometradas (en segundos) } \\ \text { [ ] } & \text { solapamiento (con numeración en subíndice si es necesario) } \\ = & \text { al final de un turno y al principio del siguiente, indican que no hay pausa audible } \\ & \text { entre ambos turnos } \\ \text { (inaud.) } & \text { fragmento inaudible } \\ \text { (palabra) } & \text { fragmento aproximado o dudoso }\end{array}$

\section{Referencias}

Acosta Ortega, L. (2017). La reparación en la interacción oral de estudiantes de ELE: comparación entre interacciones de práctica en el aula e interacciones en contextos de evaluación. Pragmática Sociocultural/Sociocultural Pragmatics, 5(2), 219-250.

Antaki, C., y Widdicombe, S. (1998). Identities as an achievement and as a tool. En C. Antaki y S. Widdicombe (Eds.), Identities in talk (pp. 1-14). London: Sage.

Bonnin, J. E. (2019). (Des)afiliación y (des)alineamiento: procedimientos interaccionales para la construcción de voz. Pragmática Sociocultural/Sociocultural Pragmatics, 7(2), 231-252. 
Bravo, D. (1999). ¿Imagen positiva vs. imagen negativa?: pragmática socio-cultural y componentes de face. Oralia, 2, 155-184.

Bravo, D. (2002). Actos asertivos y cortesía: Imagen del rol en el discurso de académicos argentinos. En M. E. Placencia y D. Bravo (Eds.), Actos de habla y cortesía en el español (pp. 141-174). Munich: LINCOM Europa.

Bucholtz, M., y Hall, K. (2005). Identity and interaction: A sociocultural linguistic approach. Discourse Studies, 7(4-5), 585-614.

Coates, J. (1997). One-at-a-time: The organisation of men's talk. En J. Coates (Ed.), Women, men and everyday talk (pp. 127-145). Basingstoke: Palgrave Macmillan.

Codó, E., Patiño Santos, A., y Unamuno, V. (2012). Hacer sociolingüística etnográfica en un mundo cambiante: Retos y aportaciones desde la perspectiva hispana. Spanish in Context, 9(2), 167-190.

De Fina, A. (2006). Group identity, narrative and self-representation. En A. De Fina, D. Schiffrin, y M. Bamberg (Eds.), Discourse and identity (pp. 351-387). New York: Cambridge University Press.

Djordjilovic, O. (2012). Displaying and developing team identity in workplace meetings - A multimodal perspective. Discourse Studies, 14(1), 111-127.

Duranti, A. (2000). Antropología lingüística. Madrid: Cambridge University Press.

Eckert, P., y McConnell-Ginet, S. (1992). Think practically and look locally: Language and gender as community-based practice. Annual Review of Anthropology, 21(1), 461-490.

Edelsky, C. (1981). Who's got the floor? Language in Society, 10(3), 383-421.

Eppler, E. (2009). Four women, two codes and one (crowded) floor: The joint construction of a bilingual collaborative floor. En P. Pichler y E. Eppler (Eds.), Gender and spoken interaction (pp. 211-234). Basingstoke: Palgrave Macmillan.

Ford, C. (2008). Women speaking up. Getting and using turns in workplace meetings. New York: Palgrave Macmillan.

Gallardo Paúls, S. (1993). La transición entre turnos conversacionales: silencios, solapamientos e interrupciones. Contextos, 11(21-22), 189-220.

Glenn, P. (2019). Conflict interaction: Insights from conversation analysis. En M. Evans, L. Jeffries, y J. O’Driscoll (Eds.), The Routledge handbook of language in conflict (pp. 215-245). New York: Routledge.

Goffman, E. (1981). Forms of talk. Philadelphia: University of Pennsylvania Press.

Goodwin, C., y Goodwin, M. H. (1992). Context, activity and participation. En P. Auer y A. Di Luzio (Eds.), The contextualization of language (pp. 77-99). Amsterdam/Philadelphia: John Benjamins Publishing Company.

Goodwin, C., y Goodwin, M. H. (2004). Participation. En A. Duranti (Ed.), A companion to linguistic anthropology (pp. 222-244). Oxford: Blackwell Publishing.

Gumperz, J. J. (1982). Discourse strategies. Oxford: Oxford University Press.

Gumperz, J. J., y Cook-Gumperz, J. (1982). Introduction: Language and the communication of social identity. In J. J. Gumperz (Ed.), Language and social identity (pp. 1-21). Cambridge: Cambridge University Press.

Hall, J. K., y Butler, E. R. (2017). The shifting role of a document in managing conflict and shaping the outcome of a small group meeting. Text $y$ Talk, 37(5), 615-638.

Hayashi, M., Raymond, G., y Sidnell, J. (2013). Conversational repair and human understanding: An introduction. En M. Hayashi, G. Raymond, y J. Sidnell (Eds.), Conversational repair and human understanding (pp. 1-40). New York: Cambridge University Press. 
Heritage, J., y Clayman, S. (2010). Talk in action: Interactions, identities, and institutions. Chichester: Wiley-Blackwell Publishing.

Hernández Flores, N. (2013). Actividad de imagen: caracterización y tipología en la interacción comunicativa. Pragmática Sociocultural, 1(2), 175-198.

Holmes, J. (2006). Workplace narratives, professional identity and relational practice. En A. De Fina, D. Schiffrin, y M. Bamberg (Eds.), Discourse and identity (pp. 166-187). New York: Cambridge University Press.

Jenks, C. J. (2007). Floor management in task-based interaction: The interactional role of participatory structures. System, 35(4), 609-622.

Jones, R., y Thornborrow, J. (2004). Floors, talk and the organization of classroom activities. Language in Society, 33(3), 399-423.

Kitzinger, C. (2012). Repair. En J. Sidnell y T. Stivers (Eds.), The handbook of conversation analysis (pp. 229-256). Oxford: Wiley-Blackwell.

Lave, J., y Wenger, E. (1991). Situated learning: Legitimate peripheral participation. Cambridge/ New York: Cambridge University Press.

Lerner, G. H. (1992). Assisted storytelling: Deploying shared knowledge as a practical matter. Qualitative Sociology, 15(3), 247-271.

Lerner, G. H. (2000). Collaborative turn sequences. En G. Lerner (Ed.), Conversation analysis. Studies from the first generation (pp. 225-256). Amsterdam/Philadelphia: John Benjamins Publishing Company.

Lerner, G. H. (2002). Turn-sharing. The choral co-production of talk-in-interaction. En C. Ford, B. A. Fox, y S. A. Thompson (Eds.), The language of turn and sequence (pp. 225-256). New York: Oxford University Press.

Liddicoat, A. J. (2007). An introduction to conversation analysis. London/New York: Continuum.

Markaki, V., y Mondada, L. (2012). Embodied orientations towards co-participants in multinational meetings. Discourse Studies, 14(1), 31-52.

Mondada, L. (2015). The facilitator's task of formulating citizens' proposals in political meetings: Orchestrating multiple embodied orientations to recipients. Gesprächsforschung-OnlineZeitschrift zur verbalen Interaktion, 16, 1-62.

Moore, E., y Nussbaum, L. (2013). La lingüística interaccional y la comunicación en las aulas. Textos de Didáctica de la Lengua y la Literatura, 63, 43-50. Recuperado de: https://www.grao.com/es/producto/revista-textos-063-julio-13-ling-isticas-y-educacionling-istica.

Pomerantz, A. (1984). Agreeing and disagreeing with assessments: Some features of preferred/ dispreferred turn shapes. En J. M. Atkinson y J. Heritage (Eds.), Structures of social action: Studies in conversation analysis (pp. 57-101). New York: Cambridge University Press.

Rampton, B. (2017). Interactional sociolinguistics. Working Papers in Urban Language y Literacies, paper 205 [online]. Recuperado de: https://research.tilburguniversity.edu/en/ publications/interactional-sociolinguistics.

Rampton, B., Maybin, J., y Roberts, C. (2015). Theory and method in linguistic ethnography. En J. Snell, S. Shaw, y F. Copland (Eds.), Linguistic ethnography. Interdisciplinary explorations (pp. 14-50). Palgrave Macmillan.

Sacks, H., Schegloff, E. A., y Jefferson, G. (1974). A simplest systematics for the organization of turn taking for conversation. Language, 50(4), 696-735.

Schegloff, E. A. (1992). On talk and its institutional occasions. En P. Drew y J. Heritage (Eds.), Talk at work: Interaction in institutional settings (pp. 101-134). New York: Cambridge University Press. 
Schegloff, E. A. (2000). Overlapping talk and the organization of turn-taking for conversation. Language in Society, 29(1), 1-63.

Schegloff, E. A. (2007). Sequence organization in interaction. A primer in conversation analysis. Vol. 1. Cambridge: Cambridge University Press.

Schegloff, E. A., Jefferson, G., y Sacks, H. (1977). The preference for self-correction in the organization of repair in conversation. Language, 53(2), 361-382.

Seedhouse, P. (2004). The interactional architecture of the language classroom. Oxford: Blackwell.

Sidnell, J. (2009). Participation. En S. D’hondt, J. O. Östman, y J. Verschueren (Eds.), The pragmatics of interaction (pp. 125-156). Amsterdam/Philadelphia: John Benjamins.

Sidnell, J., y Stivers, T. (Eds.) (2013). The handbook of conversation analysis. Chichester: John Wiley y Sons.

Szczepek, B. (2000). Functional aspects of collaborative productions in English conversation. InLiSt - Interaction and Linguistic Structures, 21. Recuperado de: http://www.inlist.unibayreuth.de/issues/21/inlist21.pdf.

Vilar, M. (2020). Interacción en reuniones de profesionales de la salud: "hacer equipo" en un hospital de Buenos Aires. [Tesis doctoral]. Facultad de Filosofía y Letras, Universidad de Buenos Aires. 